\title{
The Role of Tetramethylethylenediamine (TMEDA) in Iron Catalyzed Cross-Coupling Reactions
}

\author{
Jeffrey D. Sears, Michael L. Neidig*
}

University of Rochester

\begin{abstract}
Many iron-catalyzed cross-coupling reactions have significantly enhanced reactivity and selectivity from the use of nitrogen-based additives and supporting ligands. Tetramethylethylenediamine (TMEDA) has been shown to decrease undesirable product distributions in several carbon-carbon coupling reactions involving Grignard nucleophiles. Mechanistic investigations aimed at elucidating the role of TMEDA in iron-catalyzed cross-coupling have been limited. Bedford and coworkers concluded that while transmetallated TMEDA-ligated iron species generate cross-coupled product from $\mathrm{Mes} \mathrm{MgBr}$ and $n$ octylbromide, these species are not reactive at rates relevant to catalysis. Therefore, the reason for TMEDA suppression of undesirable reactivity such as $\beta$-hydrogen elimination remains elusive. To investigate the role of TMEDA further, a synthetic physical-inorganic approach is highly desirable. Using ${ }^{57} \mathrm{Fe}$ Mössbauer spectroscopy, EPR spectroscopy and gas chromatographic monitoring of the catalytic cross-coupling of alkenyl Grignard reagents and alkyl electrophiles, insight into the precise role of TMEDA in iron speciation and cross-coupled product distributions was obtained. Several iron-centered intermediates have been isolated and characterized using XRD. These results will provide a framework to aid in the design and development of novel iron-catalyzed cross-coupling methodologies.
\end{abstract}

